\title{
Rapid induction of complete molecular remission by sequential therapy with LDAC and sorafenib in FLT3-ITD-positive patients unfit for intensive treatment: two cases and review of the literature
}

Denise Wolleschak ${ }^{1}$, Enrico Schalk ${ }^{1}$, Christian Krogel ${ }^{1}$, Tina M Schnoeder ${ }^{1}$, Helga Luehr ${ }^{1}$, Kathleen Jentsch-Ullrich², Thomas Fischer ${ }^{1}$ and Florian $\mathrm{H}$ Heidel $^{1 *}$

\begin{abstract}
Treatment of acute myeloid leukemia remains a therapeutic challenge. Even in younger patients with a low rate of co-morbidities less than $50 \%$ of patients can be cured. For older patients or patients with significant co-morbidities, the situation appears even worse. In patients not eligible for intensive treatment approaches - e.g. due to underlying medical conditions - therapeutic approaches remain almost exclusively palliative. However, even with less intense treatment approaches, temporary remission can be achieved and this contributes to prolonged survival and improved quality of life of the respective patient. Targeted therapies have been widely used as palliative treatment in- and outside clinical trials as single agents. Combination with low-dose cytarabine (LDAC) potentially improves remission rates and can be safely administered in an outpatient setting.

Previous studies showed that additive hematologic toxicity of combinatory therapeutic approaches may arise from simultaneous treatment (e.g. chemotherapy plus targeted therapies). However, sequential therapies have already proven their feasibility in clinical trials. Here, we report two cases of rapid induction of complete molecular remission by sequential therapy with LDAC and sorafenib in patients unfit for intensive chemotherapy without significant long-term toxicity.
\end{abstract}

Keywords: AML, Sorafenib, Small molecule, LDAC, Targeted therapy

\section{Background}

Acute myeloid leukemia (AML) is an aggressive malignant disease characterized by abnormal proliferation of immature hematopoietic cells. AML is the most frequent form of acute leukemia in adults. Despite advances in chemotherapeutic treatment within the last decade, only $30-45 \%$ of patients below the age of 60 can be cured. The majority of patients, above the age of 60 , have a dismal prognosis with only $10-15 \%$ long-term survival [1-4]. While cytogenetic changes are established markers of prognosis and response $[5,6]$, several molecular markers have been defined and analyzed for their prognostic influence $[7,8]$. Length-

\footnotetext{
* Correspondence: Florian.Heidel@med.ovgu.de

'Department of Hematology and Oncology, Center of Internal Medicine, Otto-von-Guericke University Medical Center, Leipziger Str. 44, Magdeburg D-39120, Germany

Full list of author information is available at the end of the article
}

mutations (or 'internal tandem duplications', ITD) of the FLT3 tyrosine kinase occur in approximately one third of adult patients with AML [7-9]. Clinically, the occurrence of FLT3-ITD mutations in AML is associated with a higher probability of relapse and shortened disease-free- and overall-survival and therefore is considered as an unfavorable prognostic factor. FLT3-ITD mutations show a high degree of heterogeneity with respect to their length, the number of mutated clones, the allelic ratio of the duplicated segments and the insertion sites. These variables may have a dramatic impact on clinical outcome [10-14]. This view is supported by gene expression profiling demonstrating that FLT3-ITD cases are a heterogeneous entity [15].

Using myelosuppressive chemotherapy regimens, complete hematologic remission (CR) can be achieved in $60-80 \%$ of patients below the age of 60 . However, the majority of patients with AML are above the age

\section{Ciomed Central}


of 60 , and most of these patients do not qualify for or do not benefit from myelosuppressive chemotherapy. Using myelosuppressive chemotherapy, only 38-62\% of elderly patients reach $C R$ and only $5-15 \%$ show long-term overall survival compared to approximately $40 \%$ of younger patients. The prognosis is even worse for patients who are not eligible for myelosuppressive chemotherapy due to underlying medical conditions. For those who are not eligible, less aggressive treatment approaches are clearly warranted. Using low-dose cytarabine (LDAC) as monotherapy, a complete remission (CR) rate of $17 \%$ and a partial remission (PR) rate of $19 \%$ with a median survival of 15 months could be shown in a meta-analysis [16]. As this can be administered on an outpatient basis and 'reaching a remission' as well as 'outpatient treatment' are known factors to significantly improve quality of life in patients suffering from leukemia, LDAC can be considered a feasible alternative. It has been demonstrated convincingly, that outcome of LDAC treated patients is superior to hydroxyurea treatment, however this benefit seems to be restricted to cytogenetic subgroups [17]. The advantage in overall survival corresponds to the achievement of a significant remission and achievement of a CR also impacts quality of life in a positive manner. Several studies have been conducted or are under way to determine the efficacy of targeted agents in combination not only with intensive [18-20] but also low dose chemotherapy [21,22]. Sorafenib is one example of a multikinase inhibitor targeting FLT3receptor as well as BRAF, KIT and PDGFR that has been investigated as monotherapy and in various combination schedules. One important lesson learned from these trials is, that toxicity may arise from concomitant treatment with chemotherapy and targeted therapies. Combination of the FLT3 kinase inhibitor sorafenib with myelosuppressive $[23,24]$ or low-dose chemotherapy [22] with overlapping dosing schedules led to increased toxicity and required reduction of therapeutic medication. In contrast, sequential therapy of chemotherapy with kinase inhibitors has already proven its feasibility including a modest and acceptable toxicity rate [23].

Recent studies have substantially supported the concept of FLT3-ITD as a valid therapeutic target in human AML, and suggested that FLT3-ITD is capable of conferring a state of 'oncogene addiction', whereby cellular survival pathways associated with normal or precancerous cells can become hijacked, leading to a state of reliance upon key signaling molecules that can be exploited therapeutically [24]. Therefore, targeting mutated FLT3-kinase in addition to standard chemotherapy offered the opportunity to inhibit signaling pathways crucially required for maintenance of AML. A sequential therapy schedule was chosen to avoid severe hematologic toxicity as outlined above.

Here, we report on two cases of FLT3-ITD positive AML in patients unfit for intensive chemotherapy being treated with sequential therapy consisting of LDAC followed by oral administration of sorafenib resulting in rapid induction of complete molecular remission with no significant toxicity arising.

\section{Case report 1}

A 74-year old woman was referred from a county hospital to our department in January 2013 with leukocytosis, anemia and thrombocytopenia. She was in no apparent distress, however, presented on admission with reduced overall physical fitness and kachexia (consistent with an ECOGscore of 2). Laboratory findings presented as follows: Platelets (PLT) $92 \mathrm{Gpt} / \mathrm{l}$ hemoglobin (HGB) $10.6 \mathrm{~g} / \mathrm{dl}$ and white blood count (WBC) $42.30 \mathrm{Gpt} / \mathrm{l}$, with $5 \%$ neutrophils, 7\% lymphocytes, $1 \%$ eosinophils and $81 \%$ myeloid blasts. Bone marrow aspiration confirmed the replacement of normal hematopoiesis by blasts of myeloid morphology, FAB M2 (Figure 1, upper left panel). Karyotype analysis revealed a normal female karyogram $(46, \mathrm{XX})$ while molecular diagnostics was positive for an FLT3-length mutation (FLT3ITD) (Figure 2, lane 4). Her physical examination was normal except a harsh $4 / 6$ systolic murmur spreading to the carotid arteries and over the precordium. Echocardiography revealed a dilated left atrium and mild hypertrophy of the left ventricle while demonstrating good ventricular function $(\operatorname{LVEF}=65 \%)$. The aortic valve appeared highly stenotic (planimetric $0.9 \mathrm{~cm}^{2}$ ) and the mitral valve presented with a modest stenosis of $1.8 \mathrm{~cm}^{2}$ ). Taken together, our patient was not eligible for intensive, myelosuppressive chemotherapy, as the necessary volume of infusion therapy may have resulted in pulmonary edema and congestive heart failure.

Therefore, we initiated palliative treatment using LDAC at a dose of $20 \mathrm{mg}$ BID for 10 days, followed by sorafenib $400 \mathrm{mg}$ BID days 11-28 (Figure 3). The patient developed severe (grade 3-4) but transient pancytopenia of less than 7 days and received six red blood cell transfusions and three transfusions of platelets. By day 28 white blood count and platelets appeared to be normalized with regular differentiation. Bone marrow aspiration performed on day 28 (following the first cycle) showed regeneration of the hematopoiesis with slight dysplasia but complete disappearance of myeloid blasts (Figure 1, lower left panel). PCR amplified FLT3-wildtype alleles with the mutated alleles no longer detectable (Figure 2, lane 5). The patient remained in complete molecular remission with dependency on red blood cell transfusions during the LDACadministration period.

\section{Case report 2}

A collaborating rehabilitation center referred a 58-year old woman having suffered from a massive stroke of the left hemisphere with consecutive right hemiplegia and aphasia. In the past medical history, the husband 

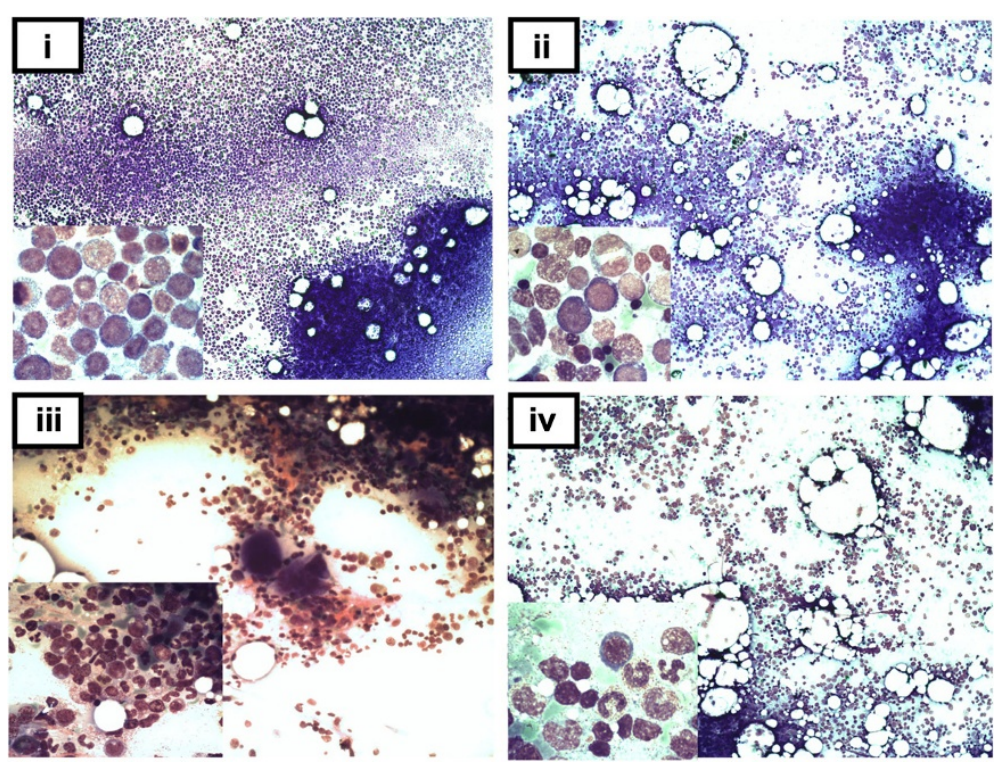

Figure 1 Cytomorphology of bone marrow (BM) aspirates at diagnosis and at remission controls obtained after the first cycle of sequential therapy. Case 1 (left column): (i) Aspirate at primary diagnosis showing 80\% BM infiltration with myeloid blasts. The malignant cells show large nuclei, narrow cytoplasm and fine azurophilic granula. (iii) Complete remission (CR1) achieved after one cycle of low-dose cytarabine (LDAC)/sorafenib. Differentiating granulopoiesis and reappearance of megakaryopoiesis as observed after reconstitution of peripheral blood counts. Case 2 (right column): (ii) Predominant expansion of the erythroid lineage with pronounced dysplasia. The non-erythroid cells show $67 \%$ of myeloid blasts consistent with the diagnosis of erythroleukemia (FAB M6). (iv) Reduction of cellularity and reconstitution of granulopoiesis following the first cycle of sequential LDAC/sorafenib therapy. Achievement of complete hematologic remission with concomitant reconstitution of peripheral blood counts.

reported heavy smoking, arterial hypertension, hyperlipoproteinemia, diabetes and overweight of his wife for several years resulting in myocardial infarction 10 years ago and consecutive congestive heart failure. The massive stroke became evident just weeks before admission. During rehabilitation blood counts were taken and revealed profound anemia. The physiatrist referred the patient to our department for red blood cell transfusion and diagnostic procedures.

On admission we saw an adipose woman with pronounced hemiplegia, aphasia (consistent with an ECOG score of 3). She appeared pancytopenic with a WBC of $1.61 \mathrm{Gpt} / \mathrm{l}$, HGB of $9.5 \mathrm{~g} / \mathrm{dl}$ and PLT count of $71 \mathrm{Gpt} / \mathrm{l}$.

Bone marrow aspiration revealed pronounced hyperplasia of erythropoiesis with severe signs of dysplasia (Figure 1, right upper panel). Among the non-erythropoietic cells we found $67 \%$ myeloid blasts, thus confirming diagnosis of acute erythroleukemia (AML FABM6). The aspirate showed a normal female karyotype $(46, \mathrm{XX})$ and a PCR positive for an FLT3-ITD mutation (Figure 2, lane 2).

In consent with the patient and her husband we initiated palliative treatment using LDAC at a dose of $20 \mathrm{mg}$ BID for 10 days, followed by sorafenib $400 \mathrm{mg}$ BID days 11-28 (Figure 3). During the first cycle of LDAC the patient remained leukopenic and developed neutropenic fever, which resolved after empiric antibiotic treatment with meropenem. Peripheral blood counts improved stepwise during the second cycle of LDAC/sorafenib and the peripheral blood counts normalized 6 weeks after treatment initiation. Bone marrow aspiration confirmed CR by cytomorphology (Figure 1, lower right panel) and complete molecular remission (Figure 2, lane 3) by PCR. The patient is currently under ongoing therapy in an outpatient setting with a relapse free survival of more than 290 days.

\section{Discussion \& review of the literature}

Currently, sorafenib is not licensed for treatment of acute myeloid leukemia (AML) but rather for treatment of hepatocellular carcinoma or renal cell carcinoma. As a consequence, sorafenib is not routinely prescribed on an outpatient basis for AML. Despite these difficulties, several reports have been published using sorafenib mostly as a palliative treatment approach for relapsed and refractory AML with a certain variability in response rates and mostly hematologic toxicity.

Sorafenib has been investigated repeatedly as a single agent for myeloid neoplasia. Within an early phase I dose escalation trial 50 patients with advanced myelodysplastic syndrome and/or refractory acute leukemia (AML, $n=48$ ) were treated with sorafenib monotherapy [25]. All patients received a starting dose of $200 \mathrm{mg}$ BID in 2 treatment arms: 


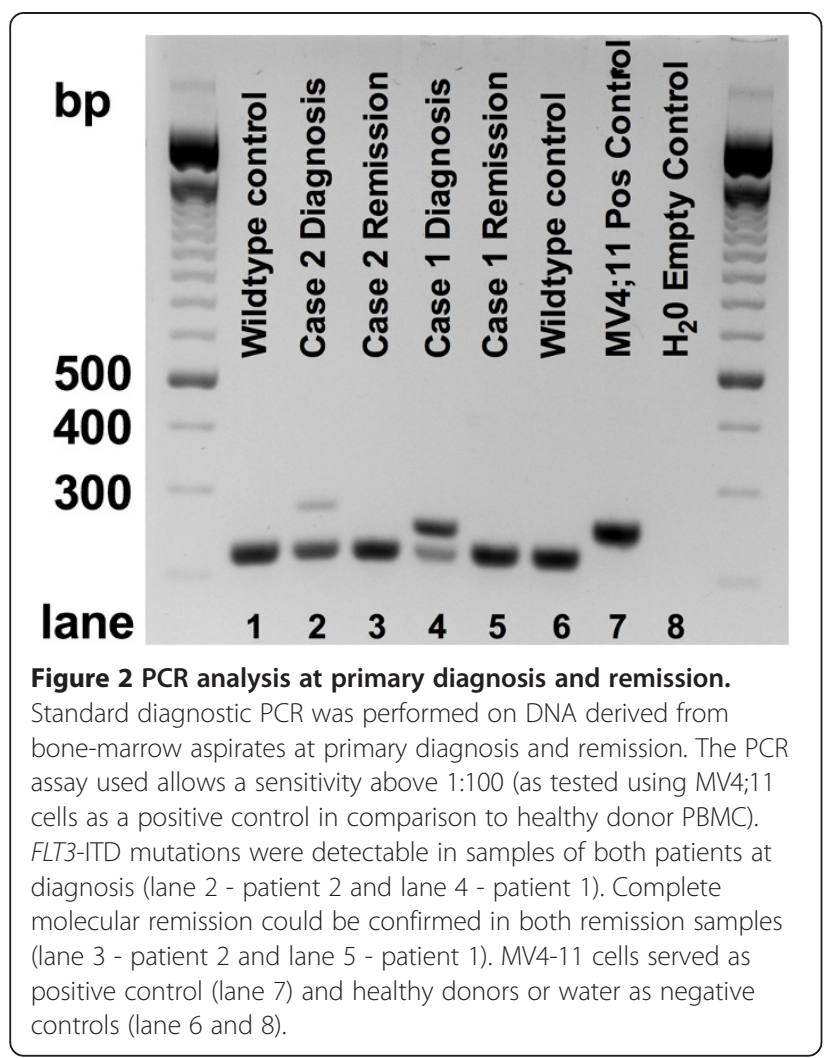

schedule (A) 5 days per week, every week for a 21 day cycle and schedule (B) for 14 days every 21 days. The maximal tolerable dose (MTD) - due to hematologic toxicity - was found to be at $400 \mathrm{mg}$ sorafenib BID. The most common side effects were fatigue (58\%), nausea/vomiting (44\%) diarrhea (36\%) and dyspnea (30\%). In terms of clinical responses, CR or CR with incomplete recovery (CRi) of platelets could be achieved in $10 \%$ of patients $(n=5)$. Moreover, 17 patients (34\%) revealed reduction of bone marrow- or peripheral blood-blast count.

Man and colleagues recruited 13 patients with relapsed or refractory FLT3-ITD positive AML to an open-label, single-arm study [26]. Patients were treated with 200 or $400 \mathrm{mg}$ sorafenib (BID) until either disease progression or eligibility for hematopoietic stem cell transplantation (HSCT). In this study, a high percentage of patients $(6 / 13$;
46\%) responded with CRi. In the context of HSCT, 65 patients with relapsed, chemotherapy-refractory FLT3-ITD positive AML have been compiled for retrospective analysis [27]. Patients were categorized in two groups with one group $(\mathrm{n}=36)$ receiving conventional chemotherapy and the second group (29 patients) receiving sorafenib as a single agent for relapsed AML after allogeneic HSCT. In 42 patients sorafenib was given at a standard dose of $400 \mathrm{mg}$ BID. Frequently reported side effects included severe pancytopenia (grade $3-4$ ) in 40/65 patients (61.5\%). Efficacy was significantly lower compared to other reports cited with $7.7 \% \mathrm{CR}, 20 \% \mathrm{CRi}$ and $44.6 \%$ of hematologic improvement/blast response. Ten patients with a complete remission achieved also a complete molecular remission (CMR). Overall response to sorafenib was comparable in a survey analysis of 29 relapsed and refractory AML patients (with a median age of 64.5 years) treated outside of clinical trials at 30 clinical centers in Germany [28]. Here, 6/23 patients (21\%) responded with CR $(n=2 ; 8.7 \%)$ or CRi $(n=4$; $17.4 \%)$. The majority of these patients had intermediate risk cytogenetics (93\%) and showed positivity for FLT3-length mutations (81\%). Less than half of the patients were NPM1-mutated (43\%). In most of the reported patients (82\%) sorafenib was given as a single agent, while $18 \%$ received sorafenib in combination with LDAC. Again, most prevalent toxicities included cytopenia (in almost every patient) followed by nausea and rash. By the time of the survey, $47 \%$ of patients were alive at a median observation of 131 days. In all other patients the median overall survival was 86 days after initiation of sorafenib treatment. Overall survival appears comparable or slightly below the results of published data on palliative treatment with either hydroxyurea or LDAC [17] in patients considered unfit for intensive chemotherapy at primary diagnosis.

Combination of LDAC with different 'small molecules' has been assessed in some clinical trials [21,22]. The combination of LDAC with sorafenib has been recently investigated in a phase I/II trial for elderly patients with AML or high-risk MDS [22]. Here, within the phase I sorafenib was started at $200 \mathrm{mg}$ BID days 2-28 followed by stepwise increase of the dose level. LDAC was given at $10 \mathrm{mg}$ s.c. BID days $1-10.13$ patients were treated in phase I. Within

\section{LDAC 20mg s.c. / BID}

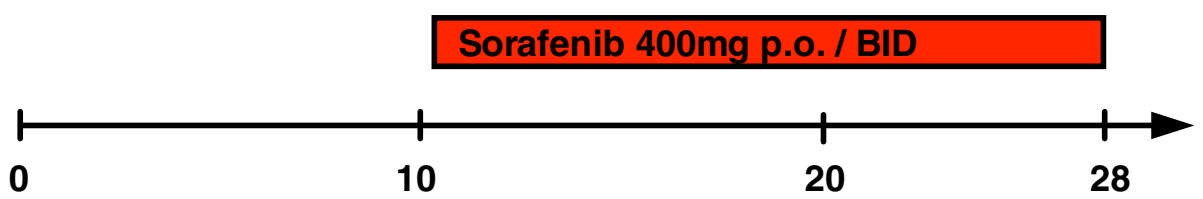

Figure 3 Treatment schedule. Sequential treatment with low-dose cytarabine (LDAC) 20 mg s.c. BID was conducted for 10 consecutive days followed by sorafenib 400 mg p.o. BID days 11-28. 
the expansion phase II, sorafenib was given at a final dose of $600 \mathrm{mg}$ days $2-28$ and LDAC $10 \mathrm{mg}$ s.c. BID days $1-10$ to another 8 patients. Out of 15 evaluable patients two patients achieved a PR (13\%) and CR (13\%), respectively, and two further patients (13\%) showed a stable disease. Given the combination of two effective drugs, the response rate seemed significantly lower than the ones published in previous reports, however, only $14 \%$ of patients revealed FLT3-ITD mutations. The most frequent non-hematologic toxicities were erythema multiforme, febrile neutropenia, hypertension, tremor and fatigue of minor severity. However, severe hematologic toxicity (grade 3-4) occurred in the vast majority of patients with $95 \%$ thrombocytopenia, $76 \%$ neutropenia and $62 \%$ anemia. This massive occurrence of hematologic toxicity led to delay or reduction of therapy and may be the consequence of increased sorafenib dosing (up to $600 \mathrm{mg}$ per day) contemporaneously administered with LDAC. Taken together, response rates using sorafenib as a single agent or in combination with low-dose chemotherapy have been reported in the range of $13-46 \% \mathrm{CR}$ or $\mathrm{CRi}$, even when applied in relapsed or refractory disease.

Previous studies have investigated the relevance of molecular monitoring of FLT3-ITD mutations. Thiede and colleagues investigated a total of 979 FLT3-ITD mutated AML patients treated with chemotherapy. Here, FLT3-ITD positive patients with high mutant/wildtype allelic ratio had a dismal prognosis in regard to overall survival (OS) and disease free survival (DFS). Moreover, these patients had a significant increased probability of relapse [12]. A second study investigated in 11 cases with AML FLT3 mutations in order to assess for minimal residual disease (MRD). MRD was measured by single step real-time PCR. Five of eleven patients developed positive MRD and all five patient MRDpositive patients experienced relapse [29]. Upon sorafenib treatment, two recent reports have assessed for residual disease on a molecular level $[27,30]$. Taken together, detection of minimal residual disease has been assessed previously, however, not systematically upon sorafenib treatment. $\mathrm{Pa}-$ tients with high mutant/wt allelic ratio treated with conventional chemotherapy seem to experience inferior overall survival (OS) and disease free survival (DFS). Positive MRD was associated with a significantly higher risk of relapse. In our hands, combined treatment with LDAC and sorafenib led to rapid induction of CMR in both patients reported. Given the available data on minimal residual disease after treatment with chemotherapy and targeted therapies, one can assume that depth of response would correlate with improved overall survival and therefore would improve quality of life. However, to the best of our knowledge, none of the previous publications investigating sorafenib for FLT3-ITD mutated AML correlated the depth of response (CMR versus $\mathrm{CHR}$ or $\mathrm{PR}$ ) with overall survival and especially quality of life. We believe that rapid induction of a
CMR could eventually contribute to prolonged survival and increased quality of life. From our perspective these questions need to be addressed prospectively in future clinical trials dealing with treatment of elderly, frail or clinically challenged patients.

The two patients reported by us, immediately achieved complete molecular remission after one cycle of sequential therapy, consistent with excellent response rates of FLT3ITD positive cohorts published before. However, in the literature responses are not exclusively restricted to FLT3mutated patients. In our hands this treatment approach appeared to be safe, feasible and could be administered to patients unfit for intensive chemotherapy in an outpatient setting. Both patients reported a clear benefit in quality of life and in both cases clinical responses were durable over several months.

Overall, we conclude that sequential therapy with LDAC followed by sorafenib appears to be an effective treatment for patients unfit for intensive chemotherapy. Sequential therapy was feasible even in an outpatient setting and did not show substantial hematologic toxicity as anticipated by simultaneous treatment approaches in recent clinical trials. However, prolonged treatment over several cycles may deserve dose reduction or shortage of treatment days to avoid hematologic toxicity, which is the major challenge of this treatment approach. A larger sample size of patients will have to be included in a prospective clinical trial to evaluate this treatment strategy in detail.

\section{Consent}

Written informed consent was obtained from both patients for publication of this case report and any accompanying images. A copy of written consent is available for review.

\section{Competing interests}

The authors declare that they have no competing interests.

\section{Authors' contributions}

All authors read and approved the final manuscript. ES and TF collected and provided data on the inpatient treatment of the cases presented. CK and KUU collected and provided data on the outpatient treatment of both patients presented. HL and TMS performed PCR analysis on the patient samples and documentation of bone marrow aspirates. DW analyzed data, compiled diagnostic data and contributed to the writing of the manuscript. $\mathrm{FHH}$ analyzed data and wrote the manuscript.

\section{Author details}

${ }^{1}$ Department of Hematology and Oncology, Center of Internal Medicine, Otto-von-Guericke University Medical Center, Leipziger Str. 44, Magdeburg D-39120, Germany. ${ }^{2}$ Private Practice for Hematology and Medical Oncology, Hasselbachplatz 2, Magdeburg D-39104, Germany.

Received: 9 April 2013 Accepted: 24 May 2013

Published: 11 June 2013

\section{References}

1. Godwin JE, Kopecky K, Head DR, Willman CL, Leith CP, Hynes HE, Balcerzak SP, Appelbaum FR: A double-blind placebo-controlled trial of granulocyte colony-stimulating factor in elderly patients with previously untreated 
acute myeloid leukemia: a southwest oncology group study (9031). Blood 1998, 91:3607-3615.

2. Goldstone AH, Burnett AK, Wheatley K, Smith AG, Hutchinson RM, Clark RE: Attempts to improve treatment outcomes in acute myeloid leukemia (AML) in older patients: the results of the United Kingdom medical research council AML11 trial. Blood 2001, 98:1302-1311.

3. Lowenberg B, Suciu S, Archimbaud E, Haak H, Stryckmans P, de Cataldo R, Dekker AW, Berneman ZN, Thyss A, van der Lelie J, et al: Mitoxantrone versus daunorubicin in induction-consolidation chemotherapy-the value of low-dose cytarabine for maintenance of remission, and an assessment of prognostic factors in acute myeloid leukemia in the elderly: final report. European organization for the research and treatment of cancer and the Dutch-Belgian hemato-oncology cooperative hovon group. J Clin Oncol 1998, 16:872-881.

4. Mayer RJ, Davis RB, Schiffer CA, Berg DT, Powell BL, Schulman P, Omura GA, Moore JO, McIntyre OR, Frei E: The cancer and leukemia group B: intensive postremission chemotherapy in adults with acute myeloid leukemia. N Engl J Med 1994, 331:896-903.

5. Mrozek K, Dohner H, Bloomfield CD: Influence of new molecular prognostic markers in patients with karyotypically normal acute myeloid leukemia: recent advances. Curr Opin Hematol 2007, 14:106-114.

6. Mrozek K, Heerema NA, Bloomfield CD: Cytogenetics in acute leukemia. Blood Rev 2004, 18:115-136.

7. Patel JP, Gonen M, Figueroa ME, Fernandez H, Sun Z, Racevskis J, Van Vlierberghe P, Dolgalev I, Thomas S, Aminova O, et al: Prognostic relevance of integrated genetic profiling in acute myeloid leukemia. $N$ Engl J Med 2012, 366:1079-1089.

8. Schlenk RF, Dohner K, Krauter J, Frohling S, Corbacioglu A, Bullinger L, Habdank M, Spath D, Morgan M, Benner A, et al: Mutations and treatment outcome in cytogenetically normal acute myeloid leukemia. N Engl J Med 2008, 358:1909-1918.

9. Frohling S, Schlenk RF, Breitruck J, Benner A, Kreitmeier S, Tobis K, Dohner H, Dohner K: Prognostic significance of activating FLT3 mutations in younger adults (16 to 60 years) with acute myeloid leukemia and normal cytogenetics: a study of the AML Study Group UIm. Blood 2002, 100:4372-4380.

10. Gale RE, Green C, Allen C, Mead AJ, Burnett AK, Hills RK, Linch DC: The impact of FLT3 internal tandem duplication mutant level, number, size, and interaction with NPM1 mutations in a large cohort of young adult patients with acute myeloid leukemia. Blood 2008, 111:2776-2784

11. Thiede C, Steudel C, Mohr B, Schaich M, Schakel U, Platzbecker U, Wermke M, Bornhauser M, Ritter M, Neubauer A, et al: Analysis of FLT3-activating mutations in 979 patients with acute myelogenous leukemia: association with $F A B$ subtypes and identification of subgroups with poor prognosis. Blood 2002, 99:4326-4335.

12. Meshinchi S, Stirewalt DL, Alonzo TA, Boggon TJ, Gerbing RB, Rocnik JL, Lange BJ, Gilliland DG, Radich JP: Structural and numerical variation of FLT3/ITD in pediatric AML. Blood 2008, 111:4930-4933.

13. Pratcorona M, Brunet S, Nomdedeu J, Ribera JM, Tormo M, Duarte R, Escoda L, Guardia R, Queipo de Llano MP, Salamero O, et al: Favorable outcome of patients with acute myeloid leukemia harboring a low-allelic burden FLT3-ITD mutation and concomitant NPM1 mutation: relevance to postremission therapy. Blood 2013, 121:2734-2738.

14. Bullinger L, Dohner K, Kranz R, Stirner C, Frohling S, Scholl C, Kim YH, Schlenk RF, Tibshirani R, Dohner H, Pollack JR: An FLT3 gene-expression signature predicts clinical outcome in normal karyotype AML. Blood 2008, 111:4490-4495.

15. Cheson BD, Simon R: Low-dose ara-C in acute nonlymphocytic leukemia and myelodysplastic syndromes: a review of 20 years' experience. Semin Oncol 1987, 14:126-133.

16. Burnett AK, Milligan D, Prentice AG, Goldstone AH, McMullin MF, Hills RK, Wheatley K: A comparison of low-dose cytarabine and hydroxyurea with or without all-trans retinoic acid for acute myeloid leukemia and highrisk myelodysplastic syndrome in patients not considered fit for intensive treatment. Cancer 2007, 109:1114-1124.

17. Fischer T, Stone RM, Deangelo DJ, Galinsky I, Estey E, Lanza C, Fox E, Ehninger G, Feldman EJ, Schiller GJ, et al: Phase IIB trial of oral Midostaurin (PKC412), the FMS-like tyrosine kinase 3 receptor (FLT3) and multitargeted kinase inhibitor, in patients with acute myeloid leukemia and high-risk myelodysplastic syndrome with either wild-type or mutated FLT3. J Clin Oncol 2010, 28:4339-4345.

18. Levis M, Ravandi F, Wang ES, Baer MR, Perl A, Coutre S, Erba H, Stuart RK, Baccarani M, Cripe LD, et al: Results from a randomized trial of salvage chemotherapy followed by lestaurtinib for patients with FLT3 mutant AML in first relapse. Blood 2011, 117:3294-3301.

19. Rollig C, Muller-Tidow C, Huttmann A, Kunzmann V, Baldus CD, Brandts C, Kramer A, Schafer-Eckart K, Neubauer A, Krause SW, et al: Sorafenib versus placebo in addition to standard therapy in adult patients $\geq 60$ years with newly diagnosed acute myeloid leukemia: results from the randomizedcontrolled soraml trial. Blood (ASH Annual Meeting Abstracts) 2012.

20. Heidel F, Cortes J, Rucker FG, Aulitzky W, Letvak L, Kindler T, Huber C, Dohner $H$, Kantarjian $H$, Fischer $T$ : Results of a multicenter phase II trial for older patients with c-kit-positive acute myeloid leukemia (AML) and high-risk myelodysplastic syndrome (HR-MDS) using low-dose ara-C and imatinib. Cancer 2007, 109:907-914.

21. Macdonald DA, Assouline SE, Brandwein J, Kamel-Reid S, Eisenhauer EA, Couban S, Caplan S, Foo A, Walsh W, Leber B: A phase I/II study of sorafenib in combination with low dose cytarabine in elderly patients with acute myeloid leukemia or high-risk myelodysplastic syndrome from the National Cancer Institute of Canada Clinical Trials Group: trial IND.186. Leuk Lymphoma 2013, 54:760-766.

22. Fischer $T$, Giles F, Paquette R, Schiller G, Ehninger G, Schiffer C, Cortes J, Kantarjian H, DeAngelo D, Heidel F, Cohen PS, Yu R, Bilic S, Zhang L, Phillips PS, Stone RM: Phase IB study of PKC412, an oral FLT3 kinase inhibitor, in sequential and simultaneous combinations with daunorubicin and cytarabine (DA) induction and high-dose cytarabine consolidation in newly diagnosed patients with AML. Haematologica 2006, 92((EHA Meeting Abstracts)).

23. Serve H, Wagner R, Sauerland C: Sorafenib in combination with standard induction and consolidation therapy in elderly AML patients: results from a randomized, placebo-controlled phase II trial. Blood (ASH Annual Meeting Abstracts) 2010, 116.

24. Smith CC, Wang Q, Chin CS, Salerno S, Damon LE, Levis MJ, Perl AE, Travers KJ, Wang S, Hunt JP, et al: Validation of ITD mutations in FLT3 as a therapeutic target in human acute myeloid leukaemia. Nature 2012, 485:260-263.

25. Borthakur G, Kantarjian H, Ravandi F, Zhang W, Konopleva M, Wright JJ, Faderl S, Verstovsek S, Mathews S, Andreeff M, Cortes JE: Phase I study of sorafenib in patients with refractory or relapsed acute leukemias. Haematologica 2011, 96:62-68.

26. Man CH, Fung TK, Ho C, Han HH, Chow HC, Ma AC, Choi WW, Lok S, Cheung AM, Eaves $C$, et al: Sorafenib treatment of FLT3-ITD(+) acute myeloid leukemia: favorable initial outcome and mechanisms of subsequent nonresponsiveness associated with the emergence of a D835 mutation. Blood 2012, 119:5133-5143.

27. Metzelder S, Wang Y, Wollmer E, Wanzel M, Teichler S, Chaturvedi A, Eilers $M$, Enghofer $E$, Neubauer A, Burchert A: Compassionate use of sorafenib in FLT3-ITD-positive acute myeloid leukemia: sustained regression before and after allogeneic stem cell transplantation. Blood 2009, 113:6567-6571.

28. Rollig C, Brandts C, Shaid S, Hentrich M, Kramer A, Junghanss C, Schleyer E, Muller-Tidow C, Berdel WE, Ritter B, et al: Survey and analysis of the efficacy and prescription pattern of sorafenib in patients with acute myeloid leukemia. Leuk Lymphoma 2012, 53:1062-1067.

29. Scholl S, Loncarevic IF, Krause C, Kunert C, Clement JH, Hoffken K: Minimal residual disease based on patient specific Flt3-ITD and -ITT mutations in acute myeloid leukemia. Leuk Res 2005, 29:849-853.

30. Al-Kali A, Cortes J, Faderl S, Jones D, Abril C, Pierce S, Brandt M, Kantarjian H, Ravandi F: Patterns of molecular response to and relapse after combination of sorafenib, idarubicin, and cytarabine in patients with FLT3 mutant acute myeloid leukemia. Clin Lymphoma Myeloma Leuk 2011, 11:361-366.

doi:10.1186/1756-8722-6-39

Cite this article as: Wolleschak et al:: Rapid induction of complete molecular remission by sequential therapy with LDAC and sorafenib in FLT3-ITD-positive patients unfit for intensive treatment: two cases and review of the literature. Journal of Hematology \& Oncology 2013 6:39. 